agents of inflammatory bowel disease in children. The prevalence of these bacteria in adult patients with IBD would give a better insight into their role as immune triggers that initiate and perpetuate chronic inflammation. This study aims to investigate the prevalence of $C$ concisus in colonic biopsy samples from adult patients with UC and compare that with control patients. Methods Adult patients who were undergoing diagnostic colonoscopy were recruited for the study, which included 68 patients with histologically proven UC and 79 control patients with no macroscopic or microscopic evidence of IBD. Biopsies were obtained during colonoscopy and immediately snap frozen in liquid nitrogen and transferred to a $-80^{\circ} \mathrm{C}$ freezer. DNA was extracted from the biopsy samples and subjected to PCR utilising Campylobacter genus-specific primers. Positive Campylobacter samples were then subjected to nested PCR using C concisus-specific primers. Fisher's exact test was used to examine any statistical difference in the presence of Campylobacter and $C$ concisus DNA between the study groups.

Results Detection of all Campylobacter DNA utilising genus specific primers was significantly higher in cases of UC, with a prevalence of $72.1 \%(49 / 68)$ compared to $24.1 \%(19 / 79)$ in control patients $(p=0.0001)$. Nested PCR for $C$ concisus DNA was positive in $30.9 \%(21 / 68)$ of biopsy samples from patients with UC, which was significantly higher than the prevalence rate of $7.6 \%(6 / 79)$ from control patients $(p=0.0005)$.

Conclusion The higher prevalence of Campylobacter genus and more specifically $C$ concisus in biopsy samples from adult patients with UC suggest a key role of this genus of bacteria in initiating the chronic inflammation that is characteristically seen in UC. To the best of our knowledge this is the first report of this association of $C$ concisus with UC in adult patients.

Competing interests None.

Keywords Campylobacter concisus, inflammatory bowel disease, ulcerative colitis.

\section{PTH-044 DETECTION OF CAMPYLOBACTER CONCISUS IN COLONIC BIOPSIES FROM ADULT PATIENTS WITH ULCERATIVE COLITIS}

doi:10.1136/gut.2011.239301.445

I Mukhopadhya, 1,* J M Thomson, ${ }^{1}$ R Hansen, ${ }^{1}$ B Alotaibi, ${ }^{1}$ S H Berry, ${ }^{1}$ E M El-Omar, ${ }^{1}$ G L Hold ${ }^{1}$ Gastrointestinal Research Laboratory, Institute of Medical Sciences, University of Aberdeen, Aberdeen, UK

Introduction The critical role of luminal bacteria in the pathogenesis of ulcerative colitis (UC) is well recognised, but an individual causative microorganism has not been singled out so far. Campylobacter concisus and other non-jejuni species of Campylobacter have been implicated as putative aetiological 